\title{
Stochastic Analysis of a Dynamics of Patterns in Condensed Matter
}

\section{$\operatorname{AUTHOR}(\mathrm{S}):$}

Ochiai, Moyuru; Yamazaki, Yoshitake; Holz, Arno; Ozao, Riko

\section{CITATION:}

Ochiai, Moyuru ...[et al]. Stochastic Analysis of a Dynamics of Patterns in Condensed Matter. 物性研究 1989, 52(4): 388-394

\section{ISSUE DATE:}

1989-07-20

URL:

http://hdl.handle.net/2433/93645

RIGHT: 


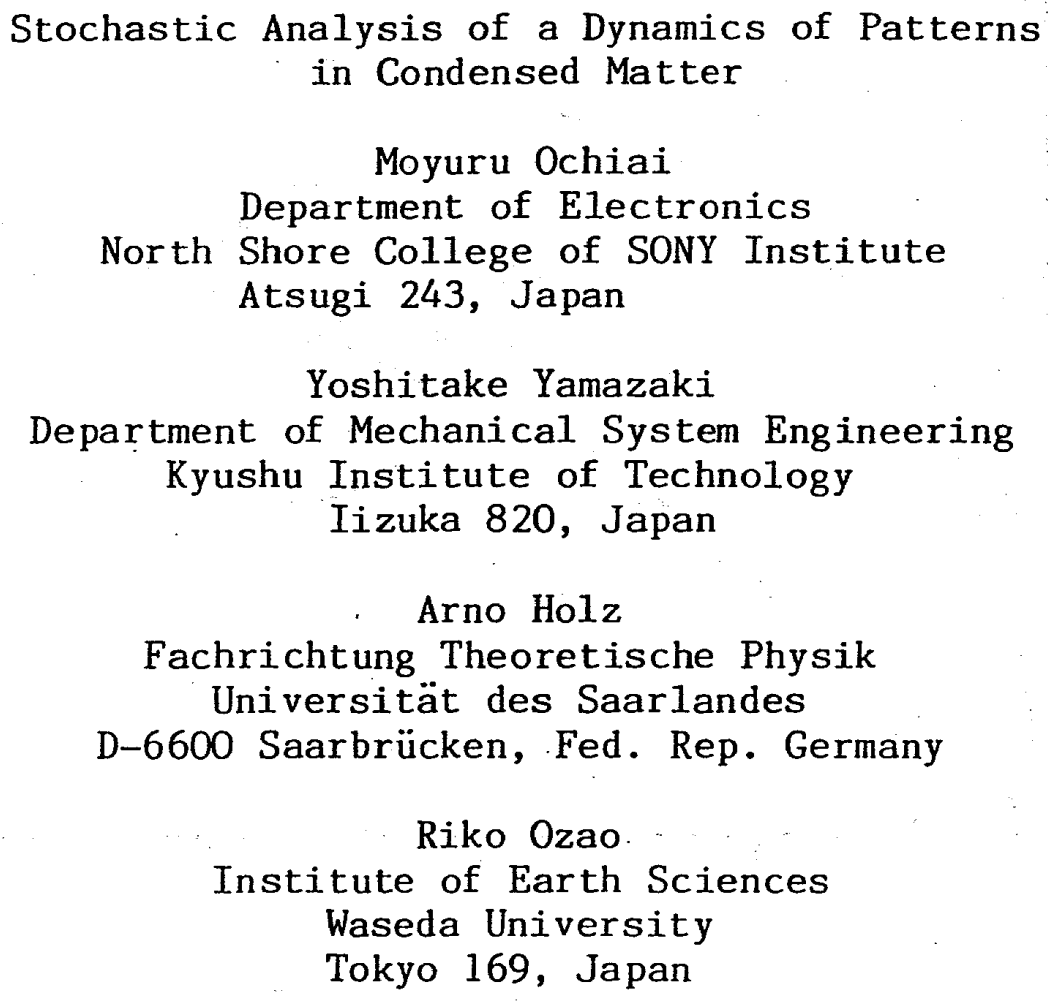

SYNOPSIS

We provide a new formulation of methods introduced in the theory of scaling expansion with the purpose of making our ideas easier and more generalized for applications.

Dynamics of patterns governed by such macroscopic laws as rate equations in case of chemical reactions and a behaviour of largely deviated fluctuations from deterministic equations describing systems far from equilibrium, are discussed.

Non-equilibrium properties of a phase transition in chemical systems are also studied. 
INTRODUCTION

In this paper, it is shown that macroscopic laws and properties of fluctuations can be easily determined by introducing a new moment generating function with a scaling expansion method which carries out coarse-graining. By introducing scaling invariants which are obtained so that macrovariables are scaled by characteristic sizes corresponding to the phenomena of interest, the cumulant BBGKY chain equation derived from the moment generating function, is automatically decoupled.

Thus, the macroscopic transport law, the equation of motion describing the fluctuating deviations from the deterministic path and the equation of the variance of fluctuations which have jointly closed forms and obey an inverted hierarchical relation, are immediately obtained.

In case of a system far from equilibrium, which, for example, stays at a critical point, the Gaussian approximation around the deterministic path becomes irrelevant. At a critical point, so called a bifurcation point, fluctuations grow up and come to play a main role $e^{[1,2]}$. Then, the usual perturbation treatment cannot be carried out. A newly determined scaling exponents of fluctuations should be introduced.

THEORY AND AN ILLUSTRATION

Making our formulation more transparent to understand, such an illustration as an autocatalytic chemical reaction is 


\section{研究会報告}

convenient.

Here, we consider the Schlögl model, of which a nonlinear rate equation has several kinds of stationary states:

$$
\begin{gathered}
A \quad \frac{k_{0}}{\gamma_{1}} \mathrm{~N} \\
\mathrm{~B}+2 \mathrm{~N} \underset{\gamma_{3}}{\stackrel{k_{2}}{\rightleftharpoons}} 3 \mathrm{~N},
\end{gathered}
$$

where the quantity of chemical species $A$ and $B$ are controlled from outside and $\mathrm{N}$ stands for the chemical intermediate.

We assume that the time evolution of the number of particles $N(t)$ of intermediate follows a Markoffian process like $N \rightarrow N+1$, $\mathrm{N} \rightarrow \mathrm{N}-1$.

Introducing the probability $P(N, t)$ which describes the number of particles $N$ of intermediate at time $t$ in the system and a moment genrating function for single variable defined as

$$
G\left(e^{i \xi}, t\right) \equiv \sum_{N=0}^{\infty} e^{i \xi N} P(N, t)
$$

we can write a master equation in the form described by a generating function as follows:

$$
\begin{gathered}
\frac{\partial G\left(e^{i \xi}, t\right)}{\partial t}=\left(e^{i \xi}-1\right)\left\{k_{0} V-\gamma_{1} e^{-i \xi} \frac{\partial}{\partial(i \xi)}+k_{2} V^{-1}\left(\frac{\partial^{2}}{\partial(i \xi)^{2}}-\frac{\partial}{\partial(i \xi)}\right)\right. \\
\left.-\gamma_{3} V^{-2} e^{-i \xi}\left(\frac{\partial^{3}}{\partial(i \xi)^{3}}-3 \frac{\partial^{2}}{\partial(i \xi)^{2}}+2 \frac{\partial}{\partial(i \xi)}\right\}\right\} G\left(e^{i \xi} ; t\right),
\end{gathered}
$$

where $k_{3} \equiv V^{-1} k_{0} A$ and $k_{2} \equiv V^{-1} k_{2} B$

Furthermore, introducing such a scaled parameter as

$$
i \xi N \equiv i \eta n
$$


「パターン形成、運動およびその統計」

we obtain a hierarchical set of moment equations which is described by only scaling invariants. First moment equation, for example, is shown by

$$
\frac{d}{d t}\langle n\rangle=k_{0}-\gamma_{1}\langle n\rangle+k_{2}\left\langle n^{2}\right\rangle-\gamma_{3}\left\langle n^{3}\right\rangle \ldots
$$

Since the equation makes a hierarchy together with the rest of higher order, it can not be closed. A solution requires the information about a behaviour of fluctuations.

At first, we should determine a scaling exponent $\nu$ of fluctuations.

\section{Putting}

$$
\mathrm{N} \equiv \mathrm{F}(\mathrm{t})+\mathrm{V}^{\nu} \mathrm{u}
$$

by the scaling assumption, we define an another generating function for single variable,

$$
G \Delta\left(e^{i \xi}, t\right) \equiv \sum_{N=0}^{\infty} e^{i \xi \Delta N} P(N, t) .
$$

where $\Delta N=N-F(t)$. Introducing a newly scaled parameter written by

$$
i \xi \Delta N \equiv i \zeta u
$$

and scaling invariants

$$
\mathrm{n}=\mathrm{f}+\mathrm{V}^{\mathcal{\nu}-1} \mathrm{u}
$$

where $f$ stands for a deterministic path, we have an equation of motion of a generating function $G_{\Delta}\left(e^{i \zeta}, t\right) \equiv G_{\Delta}$; 
研究会報告

$$
\begin{aligned}
\frac{\partial G_{\Delta}}{\partial t} & =\left\{\frac{1}{2}(i \zeta)^{2} V^{-2 \nu+1}\left(k_{0}+\gamma_{1} f+k_{2} f^{2}+\gamma_{3} f^{3}\right)\right. \\
+i \zeta & \left(-\gamma_{1}+2 k_{2} f-3 \gamma_{3} f^{2}\right) \frac{\partial}{\partial(i \zeta)} \\
+ & i \zeta V^{\nu-1}\left(k_{2}-3 \gamma_{3} f\right) \frac{\partial^{2}}{\partial(i \zeta)^{2}} \\
& \left.-i \zeta V^{2(\nu-1)} \gamma_{3} \frac{\partial^{3}}{\partial(i \zeta)^{3}}\right\} G \Delta
\end{aligned}
$$

This suggests the region of the region of the exponent $\nu$;

$$
1 / 2 \leq \nu \leq 1 .
$$

RESULTS AND DISCUSSION

If we adopt the condition $1 / 2 \leq \nu<1$, a hierarchical chain comes to the closed form;

$$
\frac{\partial f}{\partial t}=k_{0}-\gamma_{1} f+k_{2} f^{2}-\gamma_{3} f^{3} .
$$

We also attain the Fokker-Planck equation.

$$
\begin{gathered}
\frac{\partial P(u, t)}{\partial t}=\frac{1}{2} v^{1-2 \nu}\left(x_{0}+\gamma_{1} f+k_{2} f^{2}+\gamma_{3} f^{3}\right) \frac{\partial^{2}}{\partial u^{2}} P \\
-\left(-\gamma_{1}+2 k_{2} f-3 \gamma_{3} f^{2}\right) \frac{\partial}{\partial u} u P \\
-v^{-1+\nu}\left(x_{2}-3 \gamma_{3} f\right) \frac{\partial}{\partial u} u^{2} P \\
+v^{2(-1+\nu)} \gamma_{3} \frac{\partial}{\partial u} u^{3} P
\end{gathered}
$$

$-392-$ 
At a critical point, following conditions

$$
\begin{aligned}
& \dot{f}=0, \\
& (\dot{f})^{\prime}=0, \\
& (\dot{f})^{\prime \prime}=0, \\
& (\dot{f})^{\prime \prime \prime}=0
\end{aligned}
$$

Fig.1 A critical point of Schlögl model

are appropriate for a macrostate. Under the conditions, the Fokker-Planck equation comes to

$$
\begin{gathered}
\frac{\partial P(u, t)}{\partial t}=\frac{1}{2} V^{-2 \nu+1}\left\{2\left(\gamma, f+\gamma_{3} f^{3}\right)\right\} \cdot \frac{\partial^{2}}{\partial u^{2}} P(u, t) \\
+V^{2(\nu-1)} \gamma_{3} \frac{\partial}{\partial u} u^{3} P(u, t)
\end{gathered}
$$

Two terms of the RHS should be of the same order in the system size. Thus we get the scaling exponent at the critical point

$$
\nu=3 / 4 .
$$

Fluctuations at a critical point behaves as

$$
\frac{\partial\langle u\rangle}{\partial t / v^{1 / 2}} \equiv \frac{\partial\langle u\rangle}{\partial \tau}=-\gamma_{3}\left\langle u^{3}\right\rangle \text {. }
$$

This shows that the relaxation time becomes longer in $\mathrm{V}^{1 / 2}$ at a bifurcation point than at a normal stable point.

The same can be seen as follows:

Putting $f-f c \equiv \Delta f c$, from Eq. (10) we have

$$
\left(\Delta \dot{f}_{c}\right)=-\gamma_{3}\left(\Delta f_{c}\right)^{3} \quad .
$$

Starting from a small value $f_{0}$ at $t=t_{0}$, we have

$$
\Delta f_{c}=\frac{\Delta f_{0}}{\sqrt{1+2\left(\Delta f_{0}\right)^{2} \gamma_{3}\left(t-t_{0}\right)}} .
$$


研究会報告

We can thus find that $\Delta f_{c}$ approcahes to zero in the rate of $t^{-1 / 2}$ which shows a critical slowing down.

Here, we have shown how to obtain the deterministic path and determine the scaling exponent describing the system of which fluctuation does not obey the Gaussian. In particular, it has been shown much easier than usual that at a critical point the variance diverges as the $3 / 2$ power of the system size and a critical slowing down characterized by the rate of $t^{-1 / 2}$.

\section{REFERENCES}

[1] M. Ochiai: Lett. Nuovo Cimento, 40, 433 (1984)

[2] M. Ochiai, Y. Yamazaki and A. Holz: in "Dynamics of Ordering Process in Condensed Matter" (S. Komura et al. eds., Plenum Press, New York, 1988). 